\title{
Spacetime in String Theory: A Conceptual Clarification
}

\author{
Keizo Matsubara ${ }^{1}$ (D) - Lars-Göran Johansson ${ }^{1}$ \\ Published online: 25 July 2018 \\ (C) The Author(s) 2018
}

\begin{abstract}
In this paper, some conceptual issues are addressed in order to make sense of what string theory is supposed to tell us about spacetime. The dualities in string theory are used as a starting point for our argumentation. We explore the consequences of a standard view towards these dualities, namely that the dual descriptions represent the same physical situation. Given this view, one has to understand string theory in a manner such that what counts as physical spacetime is based only on the shared physical content-or common core-of the dual descriptions. In general such a spatiotemporal picture does not have to agree with, or be similar to, any of the ones suggested by naïve readings of the dual descriptions. However, in certain regimes or limits, one or the other of the initial dual descriptions may give a good effective description of physical spacetime.
\end{abstract}

Keywords Dualities $\cdot$ Empiricism $\cdot$ Semantics $\cdot$ Spacetime $\cdot$ String theory

\section{Introduction}

Philosophers have recently written a lot about dualities and their role in modern physics. There is for instance a recently published special issue of Studies in History and Philosophy of Modern Physics (Castellani and Rickles 2017a) dedicated to the topic of dualities in modern physics. ${ }^{1}$ What these dualities are will be further clarified below. Here, it suffices to state that dualities show that theoretical descriptions that appear to be very different may actually be physically equivalent.

In this paper we first review parts of the philosophical discussion on dualities. We avoid formal technicalities as much as possible and focus on the conceptual and philosophical aspects. This is for the purpose of addressing a wider range of philosophers than just philosophers of physics. A few more technical terms are introduced later in the paper, but

\footnotetext{
${ }^{1}$ See the introduction to the volume (Castellani and Rickles 2017b) for more information about the included papers. Many of the papers in the volume are referenced later in this text.
}

Keizo Matsubara

keizo.matsubara@filosofi.uu.se

Lars-Göran Johansson

lars-goran.johansson@filosofi.uu.se

1 Department of Philosophy, Uppsala University, Box 627, 75126 Uppsala, Sweden 
hopefully in such a way that the main argument can be followed even without much knowledge concerning the more intricate quantitative details.

Our main new contribution is a clarification of how one should think about spacetime in string theory. We propose a strategy for deciding what the number of spacetime dimensions would be for any given solution allowed by string theory.

We base much of our argumentation on the dualities that appear in string theory. Earlier philosophy papers have already pointed out the problems that dualities in string theory pose for traditional scientific realism. ${ }^{2}$ However, a positive account of what the consequences are for how one should conceptualize spacetime in string theory has not been sufficiently developed. ${ }^{3}$

String theory, like many other physical theories, raises questions of interpretation. What are the meanings of theoretical concepts used in such theories? When should we consider concepts used to formulate modern theories of physics to they have physical significance, as opposed to playing an auxiliary mathematical role? Questions of this kind are important for many theories, but in this paper we focus specifically on string theory. In particular we critically investigate the concept of spacetime as it is used in string theory. It is commonly claimed that if string theory is true there are 10 dimensions of spacetime, of which six dimensions are in some way hidden. ${ }^{4}$ They can for instance form a tiny compact space, or manifold, where the remaining four dimensions are the dimensions of spacetime we are already familiar with; the extra dimensions are thus "compactified". Another way in which the extra dimensions can be hidden relies on so-called "D-branes" and will be briefly discussed later in this paper. ${ }^{5}$

Our purpose is not to criticize or evaluate research in string theory; questions about its validity, truth or empirical support will not be discussed. The aim is primarily to address some semantic and conceptual issues. The view advocated is - in outline-that one should make a clear distinction between physical and mathematical claims in theoretical physics, and thus, by implication, we indicate that this distinction is not sufficiently upheld in the current discussion about string theory. In addition, we argue that the concepts used to describe the physical world must have connections to concepts used in previous theories in which they are at least indirectly connected to observations. Finding out how concepts from older established theories can be applicable in certain limits of the new theory is an important part of clarifying what the theory is supposed to tell us about reality.

How should claims about extra dimensions of spacetime be understood? Are we to understand the word "spacetime" as referring to a mathematical object, or to a physically interpreted structure? That we need more than four dimensions-in the mathematical sense-when exhibiting the mathematical structure of a physical theory is of course nothing new or peculiar to string theory. It is standard in physics to use mathematical

\footnotetext{
2 The philosophical relevance of dualities has been discussed in a number of papers. See for instance Dawid (2006, 2007), Rickles (2011, 2013a, b), Matsubara (2013) and Read (2016). In these papers it has already been discussed how dualities are problematic for traditional scientific realists. The problems that dualities pose for a straightforward spatiotemporal interpretation of the extra dimensions in string theory was also briefly discussed in Huggett and Wüthrich (2013).

3 However, some work of relevance to the issue of how to conceptualize spacetime in string theory have been done, see Huggett (2017) and De Haro (preprint).

4 The case with 10 dimensions is relevant for superstrings. Bosonic string theory requires 26 dimensions but does not contain fermions and can hence not accurately describe our world. Given the introduction of M-theory it is also sometimes claimed that spacetime is really 11-dimensional.

5 For more details see standard textbooks such as Polchinski (1998), (Becker et al. 2007) or Zwiebach (2009).
} 
spaces with a various number of dimensions. There are many examples such as the phasespaces of Hamiltonian mechanics or Hilbert spaces in quantum theory. Assuming that not any arbitrary mathematical space can be taken to represent spacetime, it is, however, pretty clear that the dimensionalities of the mathematical spaces introduced in these cases are not supposed to correspond to the dimensionality of spacetime. ${ }^{6}$ So why is string theory different in this respect? Why is it supposed that the 10-dimensional so called "target space manifolds" - in which the strings are embedded - that are needed in the formulation of string theory, represent 10-dimensional physical spacetimes? While there is a rather straightforward reason-which is described below-our argument will show that this view is problematic and cannot in general be upheld. The core issue is the relation between the mathematical formalism of string theory and its physical interpretation.

A philosophy paper discussing the nature of spacetime in the case of T-duality is Huggett (2017). In the paper Huggett argues that a target space manifold should not be understood to directly represent spacetime. In our paper the critique of target space manifolds representing spacetimes is developed further to deal also with other dualities in string theory.

We investigate the consequences of the view that dual models in string theory should be understood to be physically equivalent and describe the same physical situation. This is a view that many have already argued for, advocated or even taken for granted. We do not claim to present any new arguments for why this view on the dualities in string theory is warranted. The main new contribution of this paper is the positive articulation of how one should conceptualize spacetime in string theory if this view on the dualities is accepted.

The view expressed in this paper can be seen as a functionalist account of spacetime similar to the one expressed in $\operatorname{Knox}(2013,2017) .^{7}$ Another functionalist account of spacetime is presented in Lam and Wüthrich (2018). While the details may differ between the accounts, a common feature is that functionalist accounts of spacetime, including our own, do not require that spacetime needs to be directly given as part of the fundamental ontology of a theory. Nonetheless a functional spacetime must arise in at least some solutions of the theory, otherwise the theory would not have a chance of properly describing the world we observe around us. Our account should not be understood as antirealist about spacetime. However, what it identifies as properly spatiotemporal is not something that can be immediately read from the basic formalism. Our account will, hopefully, be further clarified from what is written in the rest of this paper. ${ }^{8}$

In Sect. 2 we give a basic survey and review of some relevant background information. In Sect. 2.1 we give an elementary outline of string theory, Sect. 2.2 contains a short description of what dualities in modern physics are, and Sect. 2.3 discusses how the dualities we are interested in are interpreted in this paper; namely in such a way that the dual descriptions represent one and the same physical situation. Previous discussions

\footnotetext{
${ }^{6}$ We are aware of the claims made by Albert (1996), who argues that the configuration space used to represent the quantum state of the universe should be seen as more physically fundamental than spacetime and perhaps is a better candidate for representing the real spacetime in some sense. This claim is in itself controversial; see for instance Wallace and Timpson (2010) for a critique. However, even if Albert would be right in claiming that the configuration space is a more physically fundamental arena than spacetime, it should not be confused with spacetime itself, as we understand the concept. Why that is so, should be apparent from the arguments presented in this paper.

7 The papers of Knox develop some ideas from Brown (2005) in a fruitful way by articulating a functionalist account of spacetime.

${ }^{8}$ For a discussion of how it can make sense to have a physical theory in which spacetime is not part of the fundamental ontology see Huggett and Wüthrich (2013).
} 
concerning the interpretation of dualities are also briefly reviewed. Readers familiar with the contents of this survey may jump ahead to Sect. 3 , where the main new ideas of this paper are articulated about spacetime in string theory based on considerations about dualities. T-duality is discussed in Sect. 3.1, mirror symmetry is discussed in Sect. 3.2 and AdS/CFT duality in Sect. 3.3. General conclusions about spacetime in string theory are presented in Sect. 3.4. In Sect. 4 we further clarify the view we have presented regarding these dualities by stressing how the dualities appear at the quantum level and the pictures suggested by the dual descriptions are artifacts of preliminary classical descriptions. Finally, Sect. 5 contains a summary of the paper and its conclusions.

\section{A Survey of Relevant Background Information}

\subsection{An Outline of String Theory}

Below follows a brief outline of string theory. For our purposes we need only a very basic and nontechnical review where we just state a number of results from string theory. ${ }^{9}$

String theory is an attempt to give a unified account of all fundamental interactions, i.e., electromagnetism, the weak and the strong nuclear force and gravity, within one theoretical framework. Since string theory is a quantum theory it is also supposed to provide us with a quantum theory of gravity. Of the four fundamental interactions only gravity has not already been successfully described in terms of quantum physics. Instead gravity is treated in terms of Einstein's general theory of relativity (GR). In GR spacetime is described in terms of a pseudo-Riemannian manifold and the metric is dynamically coupled to the matter fields via the Einstein field equations. Such a manifold can be defined with different numbers of dimensions but in traditional GR it is assumed to be four-dimensional and to represent the four-dimensional spacetime we are familiar with. ${ }^{10}$ In contrast the quantum field theories of the standard model of particles physics-which describes particles as point-like-are defined on Minkowski space. While this space is also a pseudo-Riemannian manifold it is a fixed non-dynamical background. The basic assumption of string theory is to replace the point-like particles with extended 1-dimensional strings. A unified theory must also account for both fermions and bosons and to achieve this, one uses supersymmetric string theories, or "superstring theories". There are five different superstring theories: type I, type IIA, type IIB, heterotic $\mathrm{SO}(32)$, and heterotic $E_{8} \times E_{8}$. For more details about the various string theories the reader may consult one of the standard textbooks mentioned in footnote 9 .

String theory is formulated with the use of manifolds, in which the fundamental equations of motion of 1-dimensional strings are given. The manifolds are the above mentioned target space manifolds, which are also pseudo-Riemmannian manifolds of the type used in GR to describe spacetime, albeit allowing for a different number of dimensions than the traditional four. It turns out that for quantum versions of superstring theories, consistency requires these manifolds to be 10-dimensional. To be a little bit more precise, if the string theory was formulated in a manifold with a different number of dimensions, then there would be a conformal anomaly that would lead to the theory being ill-defined.

\footnotetext{
9 Standard textbooks on string theory are Green et al. (1987), Polchinski (1998), Becker et al. (2007) and Zwiebach (2009). For an introduction to the philosophically oriented discussions on string theory see Weingard (2001) and Rickles (2008).

${ }^{10}$ See any standard textbook in general relativity, for example Wald (1984).
} 
This was generally taken to indicate that if string theory is true then the real world has a 10-dimensional spacetime. It is easy to understand why target space manifolds have been thought to represent spacetime; that was after all the reason for which the manifolds were introduced in the first place!

We critically investigate the prima facie plausible view that the 10-dimensional mathematical manifold in a straightforward manner can be said to represent a 10-dimensional physical spacetime. As will be argued, the situation is more subtle.

One example illustrating the point that it can be difficult to make sense of what is supposed to be spatiotemporal in string theory is the peculiar way in which heterotic string theories are defined. To arrive at the 10-dimensional target space in heterotic string theory a few further steps are required. Vibrations on a closed string propagating in one direction along the string are independent of those in the other. This makes it possible to treat one direction of vibrations as purely bosonic, thus preferring a 26-dimensional space to live in. The string vibrations in the other directions have the same symmetry as ordinary superstrings. For this to result in a theory of 10 dimensions, 16 of the dimensions for the bosonic direction must somehow be eliminated. This turns out to be possible in two different ways, resulting in the two string theories called heterotic $\mathrm{SO}(32)$ and heterotic $E_{8} \times E_{8}$ respectively. The extra 16 dimensions are then not interpreted as representing physical spacetime, but are considered to be internal degrees of freedom. This observation provides further motivation for why we critically discuss the topic of how to conceptualize spacetime in string theory, because it shows that there are many subtleties involved when the mathematical formalism of string theory is interpreted and that it is not obvious what in the formalism is supposed to represent spacetime.

Since we do not see any signs of the observable physical world being 10-dimensional, it was assumed that six of these dimensions are compact and very small, which explains why the dimensions do not have any currently observable consequences. Another possible explanation for why the extra dimensions stay hidden relies on so-called D-branes, which are dynamical non-perturbative entities that in the 90s were found to naturally arise in string theories. D-branes can be described as submanifolds of the target space constraining the motion of open strings. ${ }^{11}$

\subsection{What Dualities Are}

In this paper the expression "model of string theory" or equivalently "a solution of string theory", indicates a description of how the target space manifold is compactified, it can also contain other things such as background fields and D-branes. ${ }^{12}$

Specific pairs of models of theories can then be dual. Often the term "duality" is used to specify a collection of dual models of a certain type. When this is done we are talking about a type of duality, rather than a specific pair of dual models. The specific models on

\footnotetext{
11 For more details on D-branes check for instance Johnson (2003) or the standard textbooks on string theory mentioned above. That is with the exception of Green et al. (1987), which was published too early to cover D-branes.

12 Note that the use of the term "model" varies in the literature. Sometimes what we consider to be a model is regarded as a theory in its own right. Other uses of the terms "model" and "theory" can also be found. We use the term "theory" in such a way that it is applicable for the five types of superstring theories and use "model" for a specific compactification-allowing also background fields and D-branes-of one of these theories. What we mean by "model" is here basically in line with van Fraassen's use of the term where a model is a solution of a theory, see for instance van Fraassen (1980). On the other hand note that our use of the term "model" differs from the one used in De Haro (preprint).
} 
each side of the duality can be parametrized. Choosing the values of the parameters on one side of the duality means specifying a model. This model will be dual to one model on the other side of the duality; the latter model then corresponds to specific values of the parameters specifying the models defined on its side of the duality.

The statement that two models or solutions of string theory are dual implies that they give rise to the same physical spectra, scattering amplitudes, correlation functions and other measurable, or at least potentially measurable, quantities. More precisely there exists an isomorphism identifying a shared structure that is common to both models, this structure can be used to encode all the above described relevant physical data, we call this structure "the common core" of the dual models. Note that the common core may also contain things that are not measurable, that is the empirical substructure is contained in the common core but does not have to be identical with it. However, while this physically important structure is the same in both dual models the description of the models can differ quite substantially and may suggest very different pictures of reality. ${ }^{13}$

Observe that this account of dualities deals specifically with the type of information relevant for a quantum theory, such as scattering amplitudes. It is only at this quantum level that the kind of dualities we here consider appears. Polchinski (2017) describes dualities by saying that they are due to the fact that there is a quantum theory with different classical limits. While some "dualities" in the literature are not of this kind; the dualities in string theory are due to quantization. The relevant common core structure appears first when the theory is quantized, as will be further emphasized later in this paper the differences between the dual descriptions are part of the classical pictures that are used as different starting points to arrive at the quantum description.

As mentioned above there are five different supersymmetric string theories. It turns out that these theories are connected by dualities. Sometimes a duality relates two models of the same type of string theory, in other cases the duality relates models in different types of string theory. It is generally believed among string theorists that this indicates that the different versions of string theory are different limits of a more fundamental theory called "M-theory" which we do not yet understand. ${ }^{14}$ What we supposedly do know, however, is that there also exists an 11-dimensional limit of M-theory whose classical limit is 11-dimensional supergravity.

As we will see, these dualities have implications for the semantics of string theory. If dual models of string theory are just different descriptions of one and the same physical situation then one cannot take conflicting aspects of the dual descriptions at face value. One plausible response to this problem is to consider only that which is invariant under transformations from one model as having physical significance; other properties are mere mathematical auxiliaries. The view expressed in this response will be advocated in this paper. This of course applies specifically to the dual target spaces. When the target spaces are different in the dual models, this undermines our trust in them as suitable candidates to represent spacetime correctly. ${ }^{15}$

\footnotetext{
13 To find out more about dualities in string theory the reader may consult standard textbooks such as Polchinski (1998), Zwiebach (2009), Becker et al. (2007) and references therein.

${ }^{14}$ From a philosophical point of view it might be questioned whether it is warranted to conclude that there must exist an underlying M-theory. However, we will not address this question in this paper.

15 However, it can sometimes be shown that one, but not both, of the target spaces actually agrees with a good description of spacetime. That is if the relevant spacetime that agrees with one of the target spaces can be derived from the invariant structure shared by both dual models. See Huggett (2017) and the discussion later in this paper.
} 


\subsection{On the Interpretation of Dualities}

In this section we briefly review some previous work on the interpretation of dualities.

Theories in physics are formulated with the use of mathematics but are intended to be able to describe the physical world. This raises semantic questions regarding the connection between the mathematical formalism on the one hand and the theory's claims concerning physical reality on the other; what parts of the mathematical formalism are to be given a physical interpretation and how should such an interpretation be articulated? Discussions of this kind have appeared many times in philosophy of physics, for instance regarding gauge theories where the standard view is that gauge related solutions represent the same physical situation. Another example is the discussion in general relativity about the so-called "hole argument"; in this case the standard view is that many different mathematical solutions, the ones that are related by active diffeomorphisms, represent one and the same physical situation. ${ }^{16}$

As was stated above, for the account presented in this paper it is assumed that the common cores of the dual models in string theory are given one and the same physical interpretation and in addition that the shared structure or common core of the models contains everything that is even potentially measurable; even though it could also contain more structure that transcends the empirical substructure. On the other hand De Haro does not assume that duality-related models need be regarded as physically equivalent; nor does he assume that the common core of such duality-related models need be of a quantum nature as is done in this paper. ${ }^{17}$ Such a more inclusive terminology could, of course, be adopted; the term "duality" is after all used in many different ways in physics and mathematics.

Here we reserve the expression "duality" for cases where the shared common core is given one and the same physical interpretation. Nonetheless, the articles just referenced by De Haro and collaborators, agree that the view we advocate is the correct one to adopt for the dualities in string theory we are here considering. Using the terminology of De Haro et al., an internal interpretation or viewpoint is warranted in these cases and only the common core of the theories are thought to represent physical reality. To briefly explain why this is supposed to be the case: it is because the dual theories are here supposed to describe one and the same domain of the physical world. Furthermore, they are not embedded within some other theory that fixes the interpretation of the mathematical symbols; in such a situation we would have a case of an external interpretation. Thus the common core is the starting point for the interpretation and is given the same physical interpretation in both dual pictures. In addition, there is no reason to provide other extraneous parts of the dual pictures, not captured by the common core, with a physical interpretation. This means that the dual models can be seen as physically equivalent as long as it is possible to provide such an internal interpretation. ${ }^{18}$

Still, even if the common core is given the same interpretation and all potentially measurable quantities are given by the common core, some people could in principle

\footnotetext{
16 The philosophical literature on gauge theories on the one hand and the hole argument on the other is vast. As examples of useful references we suggest Healey (2007) for gauge theories and Norton (2015) for a review of the hole argument.

17 See the papers De Haro (2017), De Haro (preprint) and Dieks et al. (2015) for further details.

18 More detailed arguments for why the internal view is warranted for the dualities in string theory and a more elaborate description of what an alternative external interpretation of dualities is supposed to be can be found in De Haro (2017), De Haro (preprint) and Dieks et al. (2015).
} 
question whether this gives a full interpretation. Such a person could insist that other aspects of the dual descriptions than the common core should be given a physical interpretation. This is however not the attitude generally adopted by physicists; they tend to take for granted that dual models in string theory represent the same physical situation, at least if the duality is exact. ${ }^{19}$

To a philosopher involved in the modern debate concerning scientific realism, this attitude towards dualities might not seem to be the obvious choice. Could it not be the case that dual models instead provide us with cases of genuine underdetermination? The option of thinking about the dualities in terms of underdetermination has been considered in the philosophy literature, see for instance Rickles (2011), Matsubara (2013) and Read (2016). While these articles consider the possibility of thinking of dualities in terms of underdetermination, they also articulate views where dual models $d o$ represent the same physical situations. However, how strongly they favor the latter option differs from paper to paper.

Recently, Rickles (2017) has been less on the fence about the issue. He takes a more definite stance in favor of viewing dual formulations as representing the same underlying physical situation. Differences in descriptions should not be taken literally, the incompatibility is at the level of purely nonphysical structure. Rickles argues that dual descriptions are to be understood in the same way as different choices of gauge in gauge theories; hence they do not represent genuine alternatives. A further point stressed by Rickles is the way in which dualities are complementary in the sense that having both dual descriptions allows for practically performing calculations over a wider range of specific physical situations. This is given as a reason for not seeing the dual descriptions as being in competition. A critic could well say that the last observation is more a practical matter that should not influence the interpretative issue under discussion.

The rejection of an alternative view according to which dual models are thought of as representing genuine physical alternatives, can be seen to be motivated from the point of view of a more empiricist attitude towards theories. ${ }^{20}$ This is at least implicitly behind much of the argumentation in favor of thinking that dual models represent the same physical situation when they are not even in principle empirically distinguishable. Making the choice of only giving a physical interpretation to the common core reflects such an empiricist view on semantics. Nonetheless, this choice still allows for the physical content to include much more than what is directly observable in the sense that this is typically understood in philosophy of science. Hence, the view here presented is not an extreme empiricism or instrumentalism of the kind advocated by the logical positivists. ${ }^{21}$

We are sympathetic to this moderate kind of empiricist attitude regarding the semantics of theories. It is important to explain how the theory is supposed to connect to potential empirical measurements at least indirectly, hence the physical meaning of the formalism is tied to the context and the physical situation it is used to represent. Given that string theory is so far from being empirically supported, this makes it less clear what the theory is supposed to tell us about the world.

\footnotetext{
19 See for instance standard physics textbook discussions on dualities in Polchinski (1998), (Becker et al. 2007) and Zwiebach (2009).

20 There is also the possibility of advocating views where different attitudes are taken to different dualities depending on the specific situation. For such accounts see for instance De Haro (preprint) or Read and Møller-Nielsen (2018). However, for the purposes of the argument in this paper it is sufficient that the account we defend is the right one when it comes to the dualities in string theory that we are discussing.

21 The point that the physical content of the common core can go beyond what is directly observational in the sense used in philosophy of science has been made before, see for instance Dieks et al. (2015, 7).
} 


\section{Spacetime and Dualities}

In this section we explore the consequences of the view towards the dualities we have described above and apply it in order to articulate a positive account of how to conceptualize spacetime in string theory.

To make sense of what the common core tells us about physical reality we must understand how more familiar theories are supposed to appear in appropriate limits, so that we know when old concepts can be used as good effective descriptions of the theory. The reason for this is that the mathematical formalism by itself does not provide any theory with a physical interpretation. To articulate the physical content of the theory we would ideally like to connect the formalism more directly to empirical data and measurements. However, since string theory is yet far removed from the practical concerns of empirical data and measurements, the best we can do to spell out what the theory is supposed to mean is to clarify in which way the formalism is supposed to be related to familiar physical concepts in previous theories. This is for instance needed for identifying what is in the abstract formalism that should be interpreted as spatiotemporal properties and relations, and not merely internal degrees of freedom. We need to show how general relativitywhich is our current best theory of gravity and spacetime-gives a good approximate description in relevant solutions.

The common core is the structure shared between the dual models. The common core contains, as has been stated above, the physical spectra, scattering amplitudes, correlation functions and other measurable, or at least potentially measurable, quantities. But note again, that the common core may also contain additional structure that goes beyond the empirical substructure. Aspects differing between dual models-i.e. not part of their common cores-are thought to be surplus structure, which might be useful for mathematical purposes but should not be thought of as physically relevant. Specifically, the physical spacetime describing the physical situation must in principle be recoverable from the common core. However, this can in practice be quite difficult in general. What one can start with is to see whether or not arguments can be given to the effect that one or the other of the original classical spatiotemporal pictures associated with one or the other of the dual models actually gives a good spatiotemporal picture in the situation in question; this will however not always be the case. We will consider the implications about spacetime from T-duality, mirror symmetry and AdS/CFT duality. These dualities hold for classes of dualities between specific models, they are thus types of dualities.

\subsection{T-Duality}

In T-duality it is found that a model where the radius of a circular compactified dimension of the target space is $R$, is physically equivalent to a dual model in which the radius is $R^{\prime}=\ell_{s}^{2} / R$, where $\ell_{s}$ is the string length. The string-length is normally assumed to be comparable in size to the Planck length, which is roughly $10^{-35} \mathrm{~m} .{ }^{22}$ The model which one might prima facie take to represent a spacetime with compactified dimension of radius $R$ is found to be physically equivalent to the model which one might prima facie take to represent a spacetime with a compactified radius $R^{\prime}$. That such models can be physically equivalent shows that metric properties of the target spaces cannot naïvely be trusted to represent distances of spacetime since the distances are not invariant between the dual

22 T-duality was first discovered by Kikkawa and Yamasaki (1984). 
formulations. In the case of superstrings T-duality also changes the type of string theory, so a type IIA string theory is mapped to a type IIB string theory and vice versa. Note that in general more than one dimension can be compactified as a circle; here for simplicity we only consider one dimension to be compact and circular.

A comment often made about T-duality is that it shows that there is a minimum length in string theory. ${ }^{23}$ Thus when $R$ is below the string-length, the value of this variable is no longer interpreted as the radius of a compactified dimension; instead $R^{\prime}$ - which will now have a value higher than the string length-is taken to give the radius of spacetime. The way in which the larger of the two dual radii is the one that is taken to give the radius of spacetime fits well with the generic idea that the standard concept of distance breaks down below the Planck length. Here assuming the standard view that the string length and the Planck length are of a similar magnitude.

The argument above might have some value but it does not explain in any detail what is going on. In Huggett (2017) and Brandenberger and Vafa (1989) more detailed discussions are presented. Regardless of which dual radius we start with there is a way to, at least in principle, measure the radius of the compact dimension. The basic idea is to identify in each dual formulation the physical state that represents a photon-basically find a low energy state with the right properties to behave as a photon-and then figure out how long it would take for such a photon to return to its starting point when sent along the relevant compact dimension. ${ }^{24}$ Huggett uses the term "phenomenal space" to describe the space that is based on potential measurements of this kind. The description of the phenomenal space turns out to be in agreement with the larger radius, regardless of which of the original radii that is used for the original target space manifold. What Huggett labels "phenomenal space" is thus a good candidate for representing a real aspect of physical spacetime itself. The reason for this is because it is something that is a shared consequence of both formulations. In this paper the terms "effective space" or "effective spacetime" will also be used instead of "phenomenal space" or "phenomenal spacetime", but the terms can be used interchangeably. We stress that in this paper, it is the effective or phenomenal spacetimes we think of as being the right candidate to think of as being properly spatiotemporal; that is by playing the functional role of spacetime. While a target space manifold may give a good description of the effective or phenomenal space in some cases it is not generally true that this is the case.

To avoid any misunderstandings we point out the following. The observations above do not rule out or invalidate the use of the dual mathematical formalism where the smaller of the two numbers $R$ and $R^{\prime}$ is used to model the physical situation. The formalism based on the smaller value is just as good a starting point as the one with the larger. Here we only talk about values to emphasize that the numbers should not at this stage be uncritically interpreted as describing distances. The difference is just that in the case when one chooses the formalism using the smaller number, then the effective or phenomenal radius, which can be given a physical interpretation, will differ from the one that is prima facie suggested by the target space manifold used in that formalism. As has been pointed out above the effective or phenomenal radius always agrees with the bigger radius regardless of whether or not the formalism uses the bigger or the smaller radius.

In the simple case of T-duality we have hence found a theoretical way of describing and identifying an effective or phenomenal spacetime, whose description is shared between the

\footnotetext{
23 See for instance Polchinski (1998, 248).

${ }^{24}$ For further details please consult Huggett (2017) or Brandenberger and Vafa (1989).
} 
dual formulations. The theoretical reasoning behind this is based on considerations about possible measurements.

Already with the observations concerning T-duality we see that target spaces cannot uncritically be identified with or thought to properly represent spacetime. Even though here one of the prima facie suggested pictures happens to agree with the effective or phenomenal spacetime picture.

Based just on these observations about T-duality, the number of spacetime dimensions suggested by string theory is not put into question. There is a clear answer resolving the problem of how physical spacetime must be understood in T-duality and we are still dealing with 10 dimensions.

However, in situations when even the bigger of the two radii is very small and close to the Planck-length; we think it is justified to consider the situation to be such that it has one fewer spatial dimension. The "in principle" measurement becomes too imprecise due to quantum uncertainties. Thus effectively we have a 9 dimensional spacetime when we are dealing with cases where there is only one compact circular dimension. If more dimensions were compactified in circles - in such a way that all the circles in all dual formulations are small and of Planck length scale - then the effective spacetime would have even fewer dimensions. The disqualification of microscopic dimensions of Planck length scale as properly spatiotemporal is not stressed in Huggett (2017); that paper primarily deals with resolving the conundrum when one of the two radii is macroscopic.

The view expressed here is in line with the functional understanding of spacetime that we here advocate. What cannot even in principle be described as an effective spacetime with a suitable chronogeometric interpretation should not be counted a spatiotemporal. Our account is, as has already been stated, similar to views expressed by Knox. In Knox (2013) an expression used is "effective spacetime geometry", in Knox (2017) the account of "spacetime functionalism" is articulated. See also Brown (2005) and Lam and Wüthrich (2018) for comparison.

\subsection{Mirror Symmetry}

We now take a look at the more complicated dualities known as "mirror symmetries", in which different dual models can have target space manifolds with different topology. ${ }^{25}$

Spaces, or manifolds, with the same topology can be deformed into each other by stretching without cutting or tearing the manifold apart. Topology deals just with the overall way a space is connected, if it has holes in it for instance. A standard example is that a sphere cannot be deformed into a torus - the shape of a donut - without cutting or tearing. On the other hand the torus can be deformed into a coffee cup by stretching out a part from the torus and deforming it to the part of the cup that holds the coffee. The hole in the middle of the donut is preserved in the handle of the coffee cup.

Using the same kind of reasoning as in the T-duality case, what conclusions can be drawn regarding the effective spacetime when there is a duality of the mirror symmetry kind?

The most studied examples of mirror symmetry deal with cases where six of the dimensions of the target spaces are compactified to form so-called "Calabi-Yau

\footnotetext{
25 An early important paper on mirror symmetry is Greene and Plesser (1990). A useful review paper discussing T-duality and mirror symmetry is Giveon et al. (1994). For a comprehensive discussion on mirror symmetry from both a physical and a mathematical perspective see Hori et al. (2003).
} 
manifolds" ${ }^{26}$ In this discussion we will exclusively consider this form of mirror symmetry, but it should be noted that not all instances of mirror symmetry require that the compactified dimensions in the dual formulations are Calabi-Yau manifolds. ${ }^{27}$ It has been found that dual, and thus physically equivalent, string theory models can be defined on pairs of target spaces which are the same with regards to their non-compactified four dimensions. However, their compactified dimensions form different Calabi-Yau manifolds $\mathcal{C Y}_{1}$ and $\mathcal{C} \mathcal{Y}_{2}$ that are topologically inequivalent. Two such manifolds constitute a mirror pair. When the manifolds are exchanged, the type of string theory is also transformed so a type IIA string theory on $\mathcal{M}^{4} \times \mathcal{C Y}_{1}$ is equivalent to a type IIB string theory on $\mathcal{M}^{4} \times \mathcal{C Y}_{2}$; here $\mathcal{M}^{4}$ stands for the manifold describing the four non-compact dimensions. This is an even more drastic example compared to the T-duality case and it is even more difficult to make sense of what the spatiotemporal picture is supposed to be, because in this instance not even the topology is shared between the two dual target spaces. Furthermore, there is in contrast to the T-duality case no simple method of deriving what the relevant effective or phenomenal spatiotemporal picture would be.

Hence, if we can change certain topological properties of the compactified part of the target space manifold, while keeping the physics in the sense described above, the target spaces cannot be trusted to give even the right topology of spacetime due to the conflicting suggestions.

The relevant phenomenal or effective spacetime must be derivable from the shared common core content of the dual pictures. This means that we cannot in general accept the extra six dimensions of target space as spatiotemporal. The dual formulations agree on the number of extra dimensions, but due to the apparent conflict of different topologies there is no clear resolution of what the spatial interpretation of these extra dimensions should be, or if they should be given any physical interpretation at all. This provides a good reason why the extra six dimensions must be disqualified as being part of spacetime. It is not enough to agree on the number of dimensions if there is no clear resolution of what the spatiotemporal picture is supposed to be.

The dual formulations do, in contrast, agree on the description of the four dimensional part of the target space that is not compactified to form any Calabi-Yau manifold. Hence, this four dimensional manifold can be taken to represent a relevant effective spacetime; this is also certainly how the four dimensional manifold is traditionally interpreted in string theory. Thus, especially in cases where both Calabi-Yau manifolds are at the scale of the Planck-length - if interpreted straightforwardly-we would only consider it to be justified to acknowledge the traditional four spacetime dimensions. Note how the here given attitude of not taking descriptions that are at Planck-length scale seriously, as properly spatiotemporal, is the same as the one taken concerning ordinary T-dualities, in the case where even the bigger of the two radii is close to the Planck-length. See the end of Sect. 3.1.

\footnotetext{
26 A Calabi-Yau manifold is a complex Kähler manifold whose Kähler metric has a global holonomy contained in $S U(n)$, where $n$ is the number of complex dimensions of the manifold. There are also other equivalent definitions. Since the Calabi-Yau manifold is Kähler it is a complex manifold with a complex structure that is also endowed with compatible Riemannian and symplectic structures. This means that a Calabi-Yau space of three complex dimensions can also be seen as a six real dimensional Riemannian manifold. It is not important to understand the definition of a Calabi-Yau manifold, or the other technical details given in this footnote to follow the philosophical point made in this paper. It is enough to appreciate that mirror symmetry connects topologically inequivalent target spaces.

27 For an example where one of the mirror manifolds is not a Calabi-Yau manifold, see Gurrieri et al. (2003).
} 
While we are generally not justified in saying that there are six extra dimensions of spacetime in these solutions of string theory, it should be noted that this does not rule out that there might be extra spacetime dimensions - of the kind we are here interested in - in some cases of specific pairs of mirror symmetric models. However, to make sense of the claim that there are extra dimensions of spacetime-beyond the ones who have a common shared description-in a situation described by two dual models related by mirror symmetry, a sufficiently unique description of what the higher dimensional effective or phenomenal spacetime is must be given. This should in principle be derivable from the common core and be the same regardless of which of the pictures we use, of course this could be quite difficult to do in practice.

In some limits - that is for specific pairs of mirror manifolds - it may even be the case that one or the other of the target spaces actually gives a good description of the effective spacetime. Such a limit can be compared to the case of T-duality, in which the larger of the two radii can give a good description of that dimension as part of the effective or phenomenal spacetime; that is as long as the larger dimension is sufficiently large and not close to the Planck-length. ${ }^{28}$ If it can be shown that such a description is not possible then only the shared four-dimensional manifold would warrant a spatiotemporal interpretation. Once again we remind the reader that our understanding of spacetime is similar to views expressed in Knox $(2013,2017)$.

\subsection{AdS/CFT Duality}

To further put into question whether the number of dimensions of the mathematical target space manifold can straightforwardly be assumed to be the same as the number of dimensions of spacetime, we now take a look at another type of duality, namely the AdS/ CFT duality or correspondence.

Here "AdS" stands for "Anti-de Sitter", which is a pseudo-Riemannian maximally symmetric manifold with constant negative curvature. According to AdS/CFT duality a string theory set in AdS space is dual to a conformal field theory (CFT) on the "boundary" of that space. ${ }^{29}$ Note that the CFT is a field theory, nevertheless it turns out to be dual to a string theory. In this kind of duality, a theory set in $X$ dimensions can be physically equivalent to another in $Y<X$ dimensions. Since here two supposedly physically equivalent descriptions have different dimensionality, this feature cannot be taken at face value as reflecting something physical about the relevant effective spacetime. The most important and seminal paper on AdS/CFT is Maldacena (1998). A useful review paper on AdS/ CFT is Aharony et al. (2000).

The AdS/CFT correspondence has not been rigorously proven to be exact even though it is generally believed to be true and exact. If that is right our analysis would apply here as well; that is only the shared common core of the dual pictures should be thought of as physical. If on the other hand the duality is only approximate, a different account must be

\footnotetext{
${ }^{28}$ That one or the other of the pictures suggested by the dual formulations effectively appear in different limits is consistent with the view expressed in Polchinski (2017). In this paper he states that dualities appear when a quantum theory has multiple classical limits. What this means is basically that in the relevant limit the pair of dual quantum models are such that only one of them can be written as a small perturbation around a classical description. In such a regime, or limit, the classical picture used in the perturbative treatment can be used as a reasonably good effective description. Note however, that there may also be regimes where none of the dual models can be written as a small perturbation around a classical description.

29 The scare quotes on "boundary" above is because it is not really a boundary of the space but rather a conformal boundary; this is, however, of no relevance for the arguments made in this paper.
} 
given. See Dieks et al. (2015) and De Haro (2017) for more information about this. An interesting point made in these papers is that if the AdS/CFT duality is exact-which is the most commonly held belief-it does not make sense to treat one of the dual pictures as more fundamental and the other as emergent. Thus one should not say that there is emergence of a higher dimensional spacetime in the string theory picture from the lower dimensional boundary picture; this privileges one of the pictures in an unjustified manner. ${ }^{30}$ However, one could - as is briefly described later in this paper-discuss emergence of spacetime dimensions as a function of the parameters that specify the pair of dual models.

\subsection{Conclusions About Spacetime in String Theory}

The conclusions to draw from these dualities are that neither the specific metric nor the topology —including the dimensionality — of the mathematical target space manifold used in a specific model of string theory can be taken at face value to describe the effective or phenomenal spacetime that would appear if the model was physically realized.

The consequences of dualities in string theory fit well into a broader picture. It is commonly assumed that any quantum theory of gravity will radically change our understanding of spacetime. It is assumed that spacetime no longer can be treated as classical, i.e. as being correctly represented by a continuous manifold below the Planck-length. Hence the classical continuous description of physical spacetime in terms of pseudoRiemannian manifolds is not to be trusted at these scales. ${ }^{31}$ So thinking about quantum strings moving in continuous physical spacetime is presumably not a correct picture at a more fundamental level. This does not make it impossible to interpret some particular solutions as being approximately described in terms of an effective spacetime of the traditional kind, whereas other solutions may not allow any spatiotemporal description at all.

The discussion about dualities above is suggestive of the idea that a deeper description of string theory-for instance a future formulation of M-theory-may not use spatiotemporal concepts. Of course at present such ideas are of a rather speculative nature. ${ }^{32}$

While we allow for a future theory to be formulated without traditional spatiotemporal concepts we must require, if the theory should have any chance of describing our world, that it has at least some solutions, where to a good approximation a classical picture of the kind found in GR can be used. Here the GR-type spacetime could be obtained via some type of coarse-graining procedure. That is we have a dynamical metric field giving rise to a pseudo-Riemannian manifold, which in a sufficiently good way encodes potential measurements of distances and durations and thus plays the functional role of being spatiotemporal. $^{33}$

We furthermore suggest that we reserve the word "spacetime" to refer to that which in given a specific physical situation in a sufficiently unambiguous, albeit of course

\footnotetext{
${ }^{30}$ See also Rickles (2013a) and Teh (2013) for similar critical arguments about AdS/CFT and claims about emergence of spacetime in that context.

31 See Huggett and Wüthrich (2013) for a discussion about the various ways different approaches to quantum gravity reject the traditional spatiotemporal concepts. The paper also defends that it makes sense to have a fundamental theory, which does not rely on such classical concepts.

32 The question whether there might exist a definition of M-theory not using spatiotemporal concepts is the sixth open question in a list presented by Strominger at the meeting Strings 2014, see Strominger (2014).

33 This is similar to the view on effective or functional spacetime that is expressed in Knox (2013, 2017).
} 
approximate, way can be described by a pseudo-Riemannian manifold. With the expression "sufficiently unambiguous" we mean up to diffeomorphisms. ${ }^{34}$

We claim that the dimensionality of such a manifold corresponds to the dimensionality of spacetime, in that particular situation. Hence, it is important to find and identify this classical picture and explain how it arises from the abstract formalism. The pseudoRiemannian manifold represents the effective or phenomenal spacetime and is the basis for assigning a dimensionality to a specific physical situation, which of course may be describable by dual models.

It could be argued that demanding that the effective or phenomenal spacetime is describable by a pseudo-Riemmannian manifold is overly conservative. However, note that we do not claim that this is the fundamental and ultimate description of spacetime, it is only meant as a useful approximation. Why do we then think the dimensionality of spacetime should be decided by the dimension of the manifold used in this approximation? The reason is that we consider this a necessary requirement for connecting the new theory to our previously established spatiotemporal concepts from GR. After all, string theory must be able to reproduce the established results from GR and the standard model for it to be able to describe physical reality. Furthermore, this account provides a basis for talking about measurements of distances and durations. Without even the theoretical possibility to talk about distances and durations we do not think it is right to describe the situation as spatiotemporal.

Some might find this view overly conservative and point to the fact that many theories are conformally symmetric and only require a conformal structure for their definition. ${ }^{35}$ Such theories are scale invariant and do not change their behavior when the length scales are changed. In order to connect such a scale invariant theory to observable events-in a world where distances can be measured - the length scale must be determined. Therefore, we prefer our criterion for what should count as spatiotemporal. If only some of the theories we encounter are conformally symmetric the distances given by the pseudoRiemmannian metric will still be important. For cases where everything is conformally symmetric so that distances turn out to be physically irrelevant we find it justified to say that we do not really have a spatiotemporal physical situation. ${ }^{36}$

Given this understanding of string/M-theory, no conclusion about the number of dimensions of spacetime in our world can be drawn from the general features of the mathematical formalism since the number of dimensions of spacetime would depend on the details of the solution that is supposed to represent our world. Since the solution which describes our world is not yet identified, a conclusion about the number of spacetime dimensions in our world would be premature.

Similarly for other allowed solutions, or models, of string theory. We can ask also of counterfactual situations what the number of spacetime dimensions-in the sense here advocated-would be if such a solution was physically realized. And note that depending

\footnotetext{
34 That is we consider models where the metric has been "moved around" on the manifold under an active diffeomorphism to be equivalent since they are isometric and indistinguishable, this is a common attitude regarding how one should think about GR, see for instance Norton (2015) and references therein for further details and clarifications. In addition we could also allow for minor, not practically measurable, differences between pseudo-Riemannian manifolds of the same dimensionality that equally well perform the task.

35 Conformally symmetric theories have actions that are invariant under local rescalings of the metric.

36 The view here expressed should be understood in a way similar to how the functional role of spacetime is articulated in Knox $(2013,2017)$. Being at least in principle surveyable by rods and clocks, or measurable in terms of some other method, is part of the functional role of spacetime and this could not be achieved if we only had a conformal structure.
} 
on the details of the solution the number could vary and some solutions could even be such that they do not warrant a spatiotemporal description at all.

Here we would like to point out a difference between our account and the one presented in De Haro (preprint). In that paper it is argued that AdS/CFT-as applied in string theory-warrants an internal interpretation. Thus, according to the account given by De Haro, everything physical must be derivable from the shared common core identified in the duality; on this point we are in agreement. De Haro in the paper points to a shared mathematical structure that is common to both dual pictures, namely a 4-dimensional manifold with a conformal structure. De Haro considers this structure to represent spacetime albeit of an unfamiliar kind. According to the view advocated in this paper it does not qualify as representing spacetime since only a conformal structure is given without length scale and not a pseudo-Riemannian manifold. Despite this we could acknowledge the 4-dimensional conformal manifold to be physical, it is after all part of the common core, and perhaps be seen as a sort of proto-spacetime. While De Haro is in agreement with us that spacetime must be based on the common core, the role of the effective or phenomenal spacetime is not emphasized the way it is done in this paper. AdS/ CFT defines a class of dual models, the pairs involved are specified by values of parameters indicating the physical situation at hand. In some specific cases or limits, that is for some pairs of dual models, more relevant structure would presumably arise that can be used to describe an effective or phenomenal spacetime, of the kind we are here interested in. We will not here attempt to answer exactly when that happens, however, physicists have discussed holography and emergence of spacetime dimensions in these scenarios. Given this we take it that for some pairs of dual models-that is for certain values of the parameters specifying the pair-the higher dimensional picture would be more in agreement with the functional, effective or phenomenal spacetime we are here focusing on. ${ }^{37}$ The above comments are not in conflict with the critique, mentioned above in Sect. 3.3, that emergence should not be expressed in terms of one picture emerging from the other picture in such a way that one of the pictures is privileged. The right way of expressing it is rather that for specific pairs of dual models, the shared effective or phenomenal picture happens to agree more closely to one or the other of the pictures that are prima facie suggested by the dual descriptions. This is already a phenomenon we are familiar with from the simpler case of T-duality.

Note that in this case, as with the other dualities discussed in this paper, what we consider to have physical salience is the common core that is identified by the duality map. While it is quite surprising that this structure can arise in so different ways-by a CFT on the boundary on one hand and in terms of a string theory on the other-this is what the evidence for AdS/CFT suggests. Also, as has been discussed above, the relevant common core structure is of a quantum nature. In the next section we will further discuss how the quantum structure of string theory is derived.

\section{From Classical to Quantum}

Here we discuss how string theory is formulated as a quantum theory based on initial classical pictures. This is supposed to further clarify our account of spacetime.

\footnotetext{
37 A representative text of physicists discussing emergence of spacetime in this context is de Mello Koch and Murugan (2012), see also the philosophical papers by Rickles (2013a) and Teh (2013), they contain many additional references of this kind.
} 
String theory is a quantum theory and - as it is often the case with such theories-it was first formulated as a classical theory which was then quantized. This is reflected in the way in which string theory is typically presented in textbooks; first a classical description of strings is presented. In analogy with how a classical particle moving through spacetime traces out a world-line, a string instead traces out a two dimensional so called "worldsheet". The way the world-sheet is embedded in the target space is given by describing where the points of the world-sheet end up. From the point of view of the world-sheet this can be seen as given by a number of scalar fields defined on the world-sheet.

The classical theory thus involves a mapping of the world-sheet of strings into a target space manifold, which traditionally is considered to represent spacetime; something that we have here put into question as not always a reasonable attitude to take, at least when the theory is quantized. To turn the classical theory into a quantum theory a quantization procedure of one sort or another is needed. To fully incorporate interactions to all orders a path integral formulation can be used. ${ }^{38}$ When quantizing the string we get quantum field theories in two dimensions; that is the two dimensions of the world-sheet. These quantum field theories are conformal and two-dimensional. What is special about two-dimensional conformal field theories-compared to conformal field theories in backgrounds with a different number of dimensions - is that locally there are an infinite number of generators of conformal transformations. Two-dimensional conformal theories have also been studied outside of the context of string theory and have relevance also within statistical mechanics. A very comprehensive account can be found in Di Francesco et al. (1997). It is not important to understand the details concerning these two-dimensional field theories; the important point is just that the two-dimensional field theories on the worldsheets represent something physically more fundamental compared to the target space used in the theory. To motivate this claim one can point out that the different target spaces result in equivalent two-dimensional theories; and it is in terms of these theories the common core of the dual description can be articulated. This is to some extent a key to understanding at least some of the dualities in string theory. Witten writes the following concerning mirror symmetry:

...which is a relationship between two spacetimes that would be quite distinct in ordinary physics but turn out to be equivalent in string theory. The equivalence is possible because in string theory one does not really have a classical spacetime, but only the corresponding two-dimensional field theory; two apparently different spacetimes $X$ and $Y$ can correspond to equivalent two-dimensional field theories. (Witten 1996, 136-137, reprinted in Callender and Huggett 2001, italics added).

This understanding of string theory is today generally accepted, but what it entails concerning the nature of spacetime and its dimensionality has not been explicitly discussed as much as we think it should be.

Note the plural in the expression "spacetimes" in the quotation above. Is Witten here talking about a number of different physical worlds, with different spacetimes, or is he talking about a number of different possible mathematical representations of the same physical world? We think it is clear that only the second option makes sense, especially given what he states earlier in the quotation about there not really being a classical

38 For further details check standard textbooks on string theory such as Polchinski (1998) or Becker et al. (2007). 
spacetime. ${ }^{39}$ In other words, "spacetimes" must, to make sense of the quotation, be viewed as denoting mathematical objects, not physical ones. That means that the target space manifold is a mathematical object used in a theory formulation that should not be understood to directly represent physical spacetime itself, in accordance with what has been argued above.

To give a clarification of what is going on one can explain as follows. The setup in terms of different target space manifolds is merely a starting point for the construction of a quantized theory. After quantizing the two models with the different target spaces the quantized models will have a shared common core which defines the duality. According to the account presented in this paper, this shared common core-from which all empirical and potentially empirical consequences can be derived-is what the physical interpretation must ultimately be based on and can hence be thought of as by itself providing the relevant quantum theoretical description of the situation.

The values of the scalar fields on the two-dimensional world-sheet in string theory are normally interpreted, as mentioned above, as specifying how the string world-sheet is embedded in the target space manifold. The target-space was in string theory originally introduced for the purpose of representing spacetime. However, in the general formalism of two-dimensional conformal field theory this interpretation is not mandatory. When twodimensional conformal field theory is used in other contexts such as statistical mechanics, for instance to describe the two-dimensional Ising model, the values of the fields are of course not thought of as representing positions in spacetime. ${ }^{40}$ This just illustrates the obvious and general point that the intended physical interpretation of certain mathematical expressions is determined at least in part by the context in which these expressions are used; this has been discussed above and is something one ought to remember regardless of whether one is thinking about dualities or not.

\section{Summary and Conclusions}

We have argued that, based on the present status of string theory, there is insufficient justification for saying that string theory demands a specific number of extra dimensions of spacetime. The different dual formulations of string theory may differ in regards to both topological and metrical properties of the target space manifold. On the assumption that dual models are physically equivalent, i.e. represent the same physical situation, topological and metrical properties of the different target spaces cannot be taken at face value to represent aspects of reality.

However, we also sketch a positive account of how to conceptualize and describe spacetime in string theory. According to the view advocated in this paper the description of spacetime is supposed to be based on the shared common core of the dual models that describe the physical situation. In specific cases one or the other of the target spaces in the dual models may give an accurate description of the effective or phenomenal spacetime but in general this does not have to be the case.

We do not rule out the existence of extra physical dimensions of spacetime in the world we inhabit. We only point out that, even if it is assumed that string theory is correct, the physical status of the extra dimensions used in string theory for the solution that is

\footnotetext{
39 The quotation shows that Witten endorses the view on dualities we assume here, that is that dual descriptions are descriptions of one and the same physical scenario.

40 For further examples and details about other applications of two-dimensional conformal field theory see Di Francesco et al. (1997).
} 
supposed to represent our world is quite unclear. In our view, the number of dimensions of spacetime is dependent on the specific solution or physical situation. The dimensionality attributed to a specific solution will be the same as the dimensionality of the sufficiently unambiguous pseudo-Riemannian manifold we take to represent the effective spacetime in the physical situation described by that solution. This is an observation about solutions in general in string theory. However, one might be specifically interested in what the conclusion would be regarding the dimensionality of the world we inhabit.

We have thus suggested that one should reserve the expression "spacetime" for that part of a string theory solution which can be, albeit approximately, represented in a sufficiently unambiguous way by a pseudo-Riemannian manifold, which is such that it may be used to represent spatiotemporal relations. For a solution to be able to describe our world it must properly describe all spatiotemporal relations we can measure. In addition it might also encode some further spatiotemporal relations that are currently beyond our ability to explicitly measure. It is quite a challenge to identify what the correct manifold that describes our spacetime in the above given sense is.

To figure out the dimensionality of spacetime in our world the next step is to identify the solution of string theory that describes our world and figure out what the effective spacetime would be. This might be quite difficult since different solutions could be compatible with the observations we make and they might not agree on the number of dimensions of spacetime. Hence, to really figure out what the number of dimensions are for our spacetime, we would need both a better theoretical understanding and better empirical evidence. It is however quite possible, based on the understanding argued for in this paper, that our spacetime-in the sense used in this paper-is after all four-dimensional even if string theory happens to be a correct description of reality.

To give an example of how empirical evidence could give us stronger reasons for attributing further dimensions to our spacetime than the ones we currently observe, one may consider a situation where it is found that the extra dimensions were bigger than what was originally thought to be the case in string theory. In Arkani-Hamed et al. (1998) it was suggested that the extra dimensions could be as big as a millimeter based on the empirical measurements available at the time. ${ }^{41}$

According to Zwiebach $(2009,68)$, by 2007 experiments had ruled out extra dimensions bigger than 50 microns, and perhaps later experiments have already or will in the future decrease this limit further. But, on the other hand we might turn out to find empirical signatures that can be taken as evidence for the existence of extra dimensions of spacetime.

Finally we want to emphasize that we do not want to attack or discourage the use of the specific "pictures" used in different dual descriptions. They can be heuristically and pragmatically useful. But it is important to carefully keep in mind for which parts of such pictures we have good reasons to think they have physical significance and which we should think lack it. A well known analogy is the use of gauge variables in gauge theories; they are useful for mathematical purposes but do not represent any physical properties. Another example is how we think when we use concrete models made of balls and sticks to represent molecules. We do not think they in all respects correctly describe the molecules but they can still certainly be used to exhibit some important information.

${ }_{41}$ For details about how this could be possible see the above mentioned paper Arkani-Hamed et al. (1998). 
Acknowledgements We would like to thank Richard Dawid, Nick Huggett, James Read, Dean Rickles, Chris Smeenk, Chris Wüthrich, Eric Zaslow and the anonymous referees for helpful discussions or valuable comments on earlier versions of this manuscript. In addition we thank Riksbankens Jubileumsfond, who sponsored our work when we initially started working on this paper. KM would also like to thank The Rotman Institute of Philosophy, where some of the work on this paper was done. Finally KM thanks The John Templeton Foundation who sponsored him during his time as a Postdoctoral Research Associate at the University of Illinois at Chicago, within the project Space and Time After Quantum Gravity. Thus part of the work with this paper was performed under a collaborative agreement between the University of Illinois at Chicago and the University of Geneva and made possible by grant number 56314 from the John Templeton Foundation. Its contents are solely the responsibility of the authors and do not necessarily represent the official views of the John Templeton Foundation.

Open Access This article is distributed under the terms of the Creative Commons Attribution 4.0 International License (http://creativecommons.org/licenses/by/4.0/), which permits unrestricted use, distribution, and reproduction in any medium, provided you give appropriate credit to the original author(s) and the source, provide a link to the Creative Commons license, and indicate if changes were made.

\section{References}

Aharony, O., Gubser, S. S., Maldacena, J. M., Ooguri, H., \& Oz, Y. (2000). Large N field theories string theory and gravity. Physics Reports, 323, 183-386.

Albert, D. (1996). Elementary quantum metaphysics. In J. T. Cushing, A. Fine \& S. Goldstein (Eds.), Bohmian mechanics and quantum theory: An appraisal (pp. 277-284). Dordrecht: Kluwer Academic Publishers.

Arkani-Hamed, N., Dimopoulos, S., \& Dvali, G. R. (1998). The hierarchy problem and new dimensions at a millimeter. Physics Letters, B429, 263-272.

Becker, K., Becker, M., \& Schwarz, J. H. (2007). String theory and M-theory: A modern introduction. Cambridge: Cambridge University Press.

Brandenberger, R., \& Vafa, C. (1989). Superstrings in the early universe. Nuclear Physics B, 316, 391-410.

Brown, H. R. (2005). Physical relativity: Spacetime structure from a dynamical perspective. Oxford: Oxford University Press.

Callender, C., \& Huggett, N. (2001). Physics meets philosophy at the Planck scale. Cambridge: Cambridge University Press.

Castellani, E., \& Rickles, D. (2017a). Dualities in physics. Studies in History and Philosophy of Modern Physics, 59, 1-142.

Castellani, E., \& Rickles, D. (2017b). Introduction to special issue on dualities. Studies in History and Philosophy of Modern Physics, 59, 1-5.

Dawid, R. (2006). Underdetermination and theory succession from the perspective of string theory. Philosophy of Science, 73, 298-322.

Dawid, R. (2007). Scientific realism in the age of string theory. Physics and Philosophy, ID:11.

De Haro, S. (preprint). Spacetime and physical equivalence. http://philsci-archive.pitt.edu/12279/.

de Haro, S. (2017). Dualities and emergent gravity: Gauge/gravity duality. Studies in History and Philosophy of Modern Physics, 59, 109-125.

de Mello Koch, R., \& Murugan, J. (2012). Emergent spacetime. In J. Murugan, A. Weltman \& G. F. R. Ellis (Eds.), Foundations of space and time-reflections on quantum gravity (pp. 164-184). Cambridge: Cambridge University Press.

Di Francesco, P., Mathieu, P., \& Sénéchal, D. (1997). Conformal field theory. New York: Springer.

Dieks, D., van Dongen, J., \& de Haro, S. (2015). Emergence in holographic scenarios for gravity. Studies in History and Philosophy of Modern Physics, 52, 203-216.

Giveon, A., Poratti, M., \& Rabinovici, E. (1994). Target space duality in string theory. Physics Reports, 244, 77-202.

Greene, B. R., \& Plesser, M. R. (1990). Duality in Calabi-Yau moduli space. Nuclear Physics, B338, 15-37.

Green, M. B., Schwarz, J. H., \& Witten, E. (1987). Superstring theory (2 Volumes). Cambridge: Cambridge University Press.

Gurrieri, S., Louis, J., Micu, A., \& Waldram, D. (2003). Mirror symmetry in generalized Calabi-Yau compactifications. Nuclear Physics, B654, 61-113. 
Healey, R. (2007). Gauging what's real: The conceptual foundations of contemporary Gauge theories. Oxford: Oxford University Press.

Hori, K., Katz, S., Klemm, A., Pandharipande, R., Thomas, R., Vafa, C., et al. (2003). Mirror symmetry. Providence, RI: American Mathematical Society.

Huggett, N. (2017). Target space $\neq$ space. Studies in History and Philosophy of Modern Physics, 59, 81-88.

Huggett, N., \& Wüthrich, C. (2013). Emergent spacetime and empirical (in)coherence. Studies in History and Philosophy of Modern Physics, 44, 276-285.

Johnson, C. V. (2003). D-branes. Cambridge: Cambridge University Press.

Kikkawa, K., \& Yamasaki, M. (1984). Casimir effects in superstring theories. Physics Letters, B149, $357-360$.

Knox, E. (2013). Effective spacetime geometry. Studies in History and Philosophy of Modern Physics, 44, $346-356$.

Knox, E. (2017). Physical relativity from a functionalist perspective. Studies in History and Philosophy of Modern Physics. https://doi.org/10.1016/j.shpsb.2017.09.008.

Lam, V., \& Wüthrich, C. (2018). Spacetime is as spacetime does. Studies in History and Philosophy of Modern Physics. https://doi.org/10.1016/j.shpsb.2018.04.003.

Maldacena, J. M. (1998). The large N limit of superconformal field theories and supergravity. Advances in Theoretical and Mathematical Physics, 2, 231-252.

Matsubara, K. (2013). Realism, underdetermination and string theory dualities. Synthese, 190(3), 471-489.

Norton, J. D. (2015). The hole argument. In E. N. Zalta (Ed.). The stanford encyclopedia of philosophy. Fall 2015 edition. https://plato.stanford.edu/archives/fall2015/entries/spacetime-holearg/.

Polchinski, J. (1998). String theory (2 Volumes). Cambridge: Cambridge University Press.

Polchinski, J. (2017). Dualities of fields and strings. Studies in History and Philosophy of Modern Physics, $59,6-20$.

Read, J., \& Møller-Nielsen, T. (2018). Motivating dualities. Synthese. https://doi.org/10.1007/s11229-018$1817-5$.

Read, J. (2016). The interpretation of string theoretic dualities. Foundations of Physics, 46(2), 209-235.

Rickles, D. (Ed.). (2008). Quantum gravity: A primer for philosophers. The Ashgate companion to contemporary philosophy of physics (pp. 262-365). Aldershot: Ashgate Publishing Limited.

Rickles, D. (2011). A philosopher looks at string theory dualities. Studies in the History and Philosophy of Modern Physics, 42, 54-67.

Rickles, D. (2013a). AdS/CFT duality and the emergence of spacetime. Studies in History and Philosophy of Modern Physics, 44, 312-320.

Rickles, D. (2013b). Mirror symmetry and other miracles in superstring theory. Foundations of Physics, 43(1), 54-80.

Rickles, D. (2017). Dual theories: 'Same But Different' or 'Different But Same'? Studies in History and Philosophy of Modern Physics, 59, 62-67.

Strominger. (2014). Quantum gravity \& string theory: The past, the present and the future. Talk given at Strings 2014 in Princeton, NJ. https://mediacentral.princeton.edu/media/1_t9qjxmhz.

Teh, N. J. (2013). Holography and emergence. Studies in History and Philosophy of Modern Physics, 44, 300-311.

van Fraassen, B. C. (1980). The scientific image. Oxford: Oxford University Press.

Wald, R. M. (1984). General relativity. Chicago: The University of Chicago Press.

Wallace, D., \& Timpson, C. G. (2010). Quantum mechanics on spacetime I: Spacetime state realism. British Journal for Philosophy of Science, 61, 697-727.

Weingard, R. (2001). A philosopher looks at string theory. In C. Callender \& N. Huggett (Eds.), Physics meets philosophy at the Planck scale (pp. 138-151). Cambridge: Cambridge University Press.

Witten, E. (1996). Reflections on the fate of spacetime. Physics Today, 96(4), 24-30.

Zwiebach, B. (2009). A first course in string theory (2nd ed.). Cambridge: Cambridge University Press. 